\title{
Unerkannte Wirbelkörperfrakturen verursachen oft Rückenschmerzen
}

Wirbelkörperfrakturen bei älteren Männern bleiben meistens unerkannt - obwohl sie häufig mit neu auftretenden oder sich verschlechternden Rückenschmerzen einhergehen.

Wirbelkörperfrakturen sind die häufigste Komplikation einer Osteoporose, werden aber in der überwiegenden Zahl der Fälle nicht frühzeitig entdeckt. Männer sind in dieser Hinsicht noch schlechter dran als Frauen: Laut der Studie Osteoporotic Fractures in Men (MrOS) [1] werden bei ihnen sogar weniger als $15 \%$ aller neu aufgetretenen Wirbelkörperfrakturen diagnostiziert. Dabei sind die nicht erkannten Frakturen keineswegs durchgehend asymptomatisch. Wie eine weitere Auswertung der MrOS-Studie jetzt zeigt, machen sich Frakturen, die erst verspätet radiologisch nachgewiesen werden, vorher häufig durch Rückenschmerzen und Aktivitätseinschränkungen bemerkbar.

Bei 4396 Männern im Alter ab 65 Jahren waren zu Studienbeginn und viereinhalb Jahre später Röntgenaufnahmen von Brust- und Lendenwirbelsäule gemacht worden, gleichzeitig hatten die Studienteilnehmer Fragebögen zu Rückenschmerzen im vorausgegangenen Jahr beantwortet. Die Beurteilung von Wirbelkörperfrakturen erfolgte nach den Kriterien von Genant, die sich an der Höhenminderung orientieren (von $0=$ keine Fraktur bis $3=$ schwere Fraktur).

\section{Nur jede sechste Fraktur klinisch diagnostiziert}

Innerhalb des Untersuchungszeitraums erlitten 197 Männer mindestens eine Wirbelkörperfraktur. Eine entsprechende Diagnose erhielten in dieser Zeit aber nur 28 Patienten (0,6\%); bei 169 Patienten (3,8\%) wurde die Fraktur erst bei der abschließenden Röntgenuntersuchung festgestellt.

Über Rückenschmerzen und damit verbundene Einschränkungen berich- teten erwartungsgemäß am häufigsten Männer aus der Gruppe mit klinisch diagnostizierten Frakturen. Männer, deren Fraktur nachträglich radiologisch entdeckt wurde, waren weniger stark betroffen, hatten aber immer noch signifikant mehr Beschwerden als Männer ohne Wirbelkörperfraktur: Sie litten öfter unter Rückenschmerzen (70\% vs. $59 \%)$ und unter schweren Rückenschmerzen ( $8 \%$ vs. $4 \%$ ), sie waren häufiger (fast) immer von Rückenschmerzen geplagt ( $22 \%$ vs. $13 \%$ ) und sie waren aufgrund der Schmerzen zu einem höheren Anteil in ihren üblichen Aktivitäten eingeschränkt (34\% vs. $18 \%)$. Bei diesen Vergleichen wurden Unterschiede in Alter und anfänglichem Gesundheitszustand berücksichtigt. Auch bei Männern, die schon zu Studienbeginn über Rückenschmerzen geklagt hatten (67\%), nahm, wenn sie eine nicht erkannte Wirbelkörperfraktur erlitten, das Risiko für schwere, belastende und einschränkende Rückenschmerzen signifikant zu (um absolut $6 \%, 15 \%$ und $24 \%$ ).

\section{Auch übersehene Frakturen oft symptomatisch}

Insgesamt litten 20\% der MrOS-Teilnehmer mit zunächst nicht diagnostizierter Wirbelkörperfraktur an neuen oder sich verschlechternden Rückenschmerzen. „Das spricht dafür, dass zufällig radiologisch entdeckte Wirbelkörperfrakturen patientenrelevante Befunde sind", betonen die Studienautoren um Dr. Howard Fink vom Veterans Affairs Health Center in Minneapolis. Es sei notwendig, Wirbelkörperfrakturen frühzeitig zu erkennen, um Schmerzen, Behinderung und weitere Frakturen verhindern zu können.

\footnotetext{
Literatur

1. Fink HA et al (2017) Association of Incident, Clinically Undiagnosed Radiographic Vertebral Fractures With Follow-Up Back Pain Symptoms in Older Men: the Osteoporotic Fractures in Men (MrOS) Study. J Bone Mineral Research
}

rheuma plus $2017 \cdot 16: 121$

https://doi.org/10.1007/s12688017-0140-4

C Springer-Verlag GmbH Austria 2017

Quelle: Dr. Beate Schumacher, springermedizin.de 